\title{
Local Population Synchrony and the Encoding of Eye Position in the Primate Neural Integrator
}

\author{
Alexis Dale and ${ }^{\circ}$ Kathleen E. Cullen \\ Department of Physiology, McGill University, Montreal, Quebec, Canada, H3G 1Y6
}

Encoding horizontal eye position in the oculomotor system occurs through temporal integration of eye velocity inputs to produce tonic outputs. The nucleus prepositus is commonly believed to be the "neural integrator" that accomplishes this function through the activity of its ensemble of predominantly burst-tonic neurons. Single-unit characterizations and labeling studies of these neurons have suggested that their collective output is achieved through local feedback loops produced by direct connections between them. If this is the case, then the ensemble of burst-tonic neurons should exhibit correlated activity. To obtain electrophysiological evidence of local interactions between neurons, we simultaneously recorded pairs $(n=29)$ of burst-tonic neurons in the nucleus prepositus of rhesus macaque monkeys using eight-channel linear microelectrode arrays. We computed the magnitude of synchrony between their spike trains as a function of eye position during ocular fixations and as a function of distance between neurons. Importantly, we found that neurons exhibit unexpected levels of positive synchrony, which is maximal during contralateral fixations and weakest when neurons are located far apart from one another $(>300 \mu \mathrm{m})$. Together, our results support a role for shared inputs to ipsilateral pairs of burst-tonic neurons in the encoding of eye position in the primate nucleus prepositus.

Key words: correlations; electrophysiology; prepositus; simultaneous recordings

\section{Introduction}

The nucleus prepositus is the mammalian oculomotor horizontal velocity-to-position neural integrator (Robinson, 1989; Moschovakis, 1997; Goldman et al., 2002). It receives inputs from structures that control saccades, pursuit, and gaze-stabilizing reflexes (for review, see McCrea and Horn, 2006) and, in turn, sends direct projections to the extraocular motor nuclei. Notably, the responses of nucleus prepositus neurons reflect the dynamic characteristics of the motor plant (monkey: McCrea and Cullen, 1992; McFarland and Fuchs, 1992; Green et al., 2007; cat: LopezBarneo et al., 1982; Delgado-García et al., 1989), leading to the proposal that its widespread projections distribute a central representation or "efference copy" (von Holst and Mittelstadt, 1950) of horizontal eye movement to the oculomotor system and higher-order centers involved in spatial orientation (cat: McCrea and Baker, 1985b).

The nucleus prepositus is commonly known as the "oculomotor neural integrator" because it essentially performs a temporal integration of its eye velocity input to generate a sustained eye position signal. Although intrinsic cellular mechanisms, such as

\footnotetext{
Received Sept. 30, 2014; revised Jan. 21, 2015; accepted Feb. 5, 2015.

Author contributions: A.D. and K.E.C. designed research; A.D. performed research; A.D. analyzed data; A.D. and K.E.C. wrote the paper.

This study was supported by the Canadian Institutes for Health Research. We thank D. Mitchell, M. Jamali, J. Carriot, and Y. S. Wong for critically reading this manuscript, W. Kucharski for mechanical expertise, and S. Nuara for animal care assistance.

The authors declare no competing financial interests.

Correspondence should be addressed to Dr. Kathleen E. Cullen, Mclntyre Medical Building, Room 1219, 3655 Promenade Sir William 0sler, Montreal, Quebec, Canada, H3G 1Y6. E-mail: kathleen.cullen@mcgill.ca.

DOI:10.1523/JNEUROSCI.4253-14.2015

Copyright $\odot 2015$ the authors $\quad 0270-6474 / 15 / 354287-09 \$ 15.00 / 0$
}

active membrane properties, likely contribute to the sustained tonic firing (Delgado-García et al., 2006), models of the mammalian oculomotor neural integrator typically also include a system of reciprocal connections between burst-tonic neurons (Galiana and Outerbridge, 1984; Cannon and Robinson, 1985; Arnold and Robinson, 1997). This latter idea is supported by findings that neurons send excitatory collaterals to other neurons in the nucleus prepositus on the same side (for review, see McCrea and Horn, 2006). Theoretically, because neurons encoding similar information project back onto one another, their input signal is effectively perpetuated. If the nucleus prepositus uses this mechanism for integration, then the activities of ipsilateral pairs of neurons should be related positively with a peak correlation at near-zero latencies. To date, the questions of whether pairs of neurons in the nucleus prepositus display synchronous activity, and if so what role this might have in encoding eye position, have not been addressed.

Accordingly, here we made simultaneous recordings from pairs of neurons in the primate nucleus prepositus. On average, correlations on a $1 \mathrm{~ms}$ timescale were significant with zero lag and greater than predicted by the fact that neurons similarly encode eye position. However, our analysis further revealed that the robustness of this excess synchrony decreased with increasing ipsilateral eye position, suggesting that neuronal activity was actually the least synchronized when the neurons were most active. Notably, the strength of the correlation also significantly decreased with increasing distance between neurons. This mechanism has analogies with the fish homolog of the nucleus prepositus, in which positive correlations between neuron pairs also vary with eye position (Aksay et al., 2003) and are strongest between nearby neurons (Miri et al., 2011). The temporal resolution of our re- 
cordings also enabled us to further this latter result by revealing the existence of millisecond synchrony between neighboring neuron pairs. Thus, overall, our present results provide new insight into the global functional connectivity of the primate nucleus prepositus by demonstrating behaviorally dependent changes in synchrony during coding of eye position that likely reveal a strategy common across vertebrates.

\section{Materials and Methods}

Experimental procedures outlined below were approved by the McGill University Animal Care Committee and are in compliance with the Canadian Council on Animal Care guidelines.

Surgical procedures. Two rhesus monkeys (Macaca mulatta, one male one female) underwent aseptic surgery in preparation for acute extracellular recordings. The details of surgical treatment were identical to those described previously (Dale and Cullen, 2013): under surgical levels of isoflurane anesthesia $(0.8-1.5 \%)$, monkeys were implanted with a stainless steel head post and a recording chamber positioned with a $30^{\circ}$ posterior angle and a $26^{\circ}$ lateral angle over the nucleus prepositus. The implants were chronically secured to each animal's skull with stainless steel cortical screws and dental acrylic. In addition, each monkey was implanted with an $18 \mathrm{~mm}$ eye coil (three loops of Teflon-insulated stainless steel wire) behind the conjunctiva of one eye (Fuchs and Robinson, 1966). Monkeys received buprenorphine $(0.01 \mathrm{mg} / \mathrm{kg}$, i.m.) as postoperative analgesia, and the antibiotic cefazolin $(25 \mathrm{mg} / \mathrm{kg})$ was injected twice daily for $10 \mathrm{~d}$. Animals were given at least 2 weeks to recover before beginning experiments.

Behavioral paradigms. Throughout recordings, monkeys were seated, head restrained, in a primate chair fixed to a vestibular turntable in the center of a $1 \mathrm{~m}^{3}$ magnetic field coil (CNC Engineering). Monkeys were trained, using juice reward, to look at a red helium-neon laser that projected via two galvanometer controlled mirrors onto a cylindrical screen located $55 \mathrm{~cm}$ away from their eyes. The position of the target was controlled through REX, a QNX-based real-time data acquisition system (Hayes et al., 1982).

To elicit saccades and periods of fixation, the laser target was stepped randomly to horizontal positions between -30 and $30^{\circ}$. Neuronal responses were also confirmed during smooth pursuit eye movements (sinusoidal target motion, $0.5 \mathrm{~Hz}$; peak velocity, $\pm 40 \% \mathrm{~s}$ ) and eye movements driven by the vestibulo-ocular reflex during whole-body rotations $(0.5 \mathrm{~Hz}, \pm 40 \% \mathrm{~s})$ in the dark.

Data acquisition. The nucleus prepositus was targeted relative to the abducens nucleus, which was identified by the typical "singing beehive" sound of its discharge during spontaneous eye movements (Fuchs and Luschei, 1970; Robinson, 1970). All of the neurons recorded in this study were isolated at sites at least $2 \mathrm{~mm}$ posterior to the abducens nucleus, which ensured that they were located outside this nucleus. Extracellular single-unit activity of burst-tonic neurons consistent with that previously characterized in the monkey nucleus prepositus (McCrea and Cullen, 1992; McFarland and Fuchs, 1992; Cullen et al., 1993; Sylvestre et al., 2003; Dale and Cullen, 2013) was recorded using a linear microelectrode array (LMA; MicroProbes) with eight 12.5- $\mu \mathrm{m}$-diameter recording sites $(\sim 1 \mathrm{M} \Omega$ impedance) spaced $50 \mu \mathrm{m}$ apart (Figs. 1A1,B1). Signals from each recording site were bandpass filtered from $300 \mathrm{~Hz}$ to $3 \mathrm{kHz}$ and sampled on distinct data channels at $30 \mathrm{kHz}$. Gaze and head position were measured continuously using the magnetic search coil technique (Fuchs and Robinson, 1966; Judge et al., 1980), low-pass filtered at 250 $\mathrm{Hz}$, and acquired at $1 \mathrm{kHz}$. Eight channels of neural data plus all behavioral data were collected through the Cerebus Neural Signal Processor (Blackrock Microsystems).

After recordings, each channel of neural data was imported into Offline Sorter (Plexon) for channel-by-channel analyses. For each channel with an isolated neuron, high-pass-filtered $(300 \mathrm{~Hz})$ voltage traces were marked at a positive (or negative) threshold and snipped in a $700 \mu \mathrm{s}$ window around each threshold crossing to obtain characteristic waveforms of the action potentials of the neuron. These waveforms were then aligned on their voltage peak (or trough) and sorted via the proprietary principal components analysis method of the software into clusters cor- responding to the neuron(s) and background activity. The time stamps associated with the waveforms of a given neuron were later used to generate a binary $(0$, no spike; 1 , one spike) vector of the unit activity of the neuron with a $1 \mathrm{~ms}$ bin width (UA).

Data analysis. Neural and behavioral data were analyzed in the MATLAB (MathWorks) programming environment. Behavioral signals were digitally filtered at $125 \mathrm{~Hz}$, and then an eye position signal was generated by computing the difference between gaze and head position. Eye, gaze, and head-position signals were then digitally differentiated to obtain velocity signals. An estimated firing rate signal for each neuron was also generated by convolving a Gaussian function ( $5 \mathrm{~ms}$ SD for assessment of bursts, 10 $\mathrm{ms}$ for fixation and smooth eye movement analysis) with the unit activity.

Burst-tonic neurons were initially selected for increased firing rate during ipsilaterally directed eye position and eye velocity. Saccadic eye movements were isolated using a $20 \%$ s velocity threshold. To determine the eye position sensitivity of each neuron, we pooled epochs of headfixed, ocular fixation lasting at least $300 \mathrm{~ms}$ and that began at least $50 \mathrm{~ms}$ after the offset and ended at least $50 \mathrm{~ms}$ before the onset of a spontaneous saccade. Horizontal eye-position sensitivities are reported as the slope of a linear regression between mean firing rate of a given epoch and the fixation position of that epoch over at least 40 fixations (Figs. 1A2,B2). The correlation coefficient between the mean firing rates of two neurons across all fixations was computed as signal correlations (Fig. 2A).

To specifically investigate local connections between neurons in the nucleus prepositus, we performed all analyses reported in Results on neuronal responses during periods of head-fixed ocular fixation. In our analysis, responses were binned as a function of the corresponding eye position in $5^{\circ}$ bins centered at 0 and $\pm 5,10,15$, and $20^{\circ}$.

The cross-correlation between two neurons was calculated using the MATLAB function $x$ corr as follows:

$$
C_{1}(\tau)=\sum_{t} \mathrm{UA}_{1, a}(t) * \mathrm{UA}_{2, a}(t-\tau) * \frac{1}{T_{a}-|\tau|},
$$

where $\mathrm{UA}_{1}$ and $\mathrm{UA}_{2}$ correspond to the unit activities of the neurons, and $\tau$ is measured in $1 \mathrm{~ms}$ windows. The subscript $a$ denotes the fixation epoch from which the unit activity is being used, $T_{a}$ is the length of the fixation epoch (here, we truncated each fixation to be of equal length, so $T$ is always $300 \mathrm{~ms}$ ), and the final term scales to give a result that is unbiased to the amount of data across all lags (unbiased option in MATLAB). We calculated the level of synchronous activity between the two neurons as $C_{1}(0)$ (Fig. $2 B$, top, left side). Note that we ensured that both neurons were firing in all fixation epochs analyzed for each fixation bin. Also, the labeling of neuron 1 and neuron 2 was chosen in order of LMA channel numbering and Offline Sorter unit numbering, and the same analysis could have been computed with the neuron labels reversed.

We used the shuffle predictor approach (Perkel et al., 1967) to compute an estimate of the synchrony between two neurons that is not attributable to their typical firing patterns. First, the raw cross-correlation between the two neurons was computed using Equation 1 for each epoch in a given fixation bin and taking the average cross-correlation over all epochs. Next, we calculated an estimate of the probable or "expected" level of correlations by computing the cross-correlation using shuffled fixation epochs (Perkel et al., 1967):

$$
C_{2}(\tau)=\sum_{t} \mathrm{UA}_{1, a}(t) * \overline{\mathrm{UA}_{2, b}(t-\tau)} * \frac{1}{T-|\tau|},
$$

where $T=300 \mathrm{~ms}$, and the subscript $b$ corresponds to a resampled (without replacement) sequence of the epochs within the same fixation bin. Note that Equations 1 and 2 differ only in the subscript $b$ in place of $a$ corresponding to epoch $b$ from the second neuron that is being compared with epoch $a$ from the first neuron (Fig. 2B, right side). By calculating the average and $\mathrm{SD}$ of $C_{2}(0)$ for shuffled epochs across 100 independent shuffle operations, we achieved an estimated range for the expected level of synchrony between the two neurons for that fixation bin (Fig. 2B, bottom). Finally, to compute the "unexpected" coincident spikes between the two neurons, we found the difference between the average raw cross-correlation and the average shuffled cross-correlation. 
A1

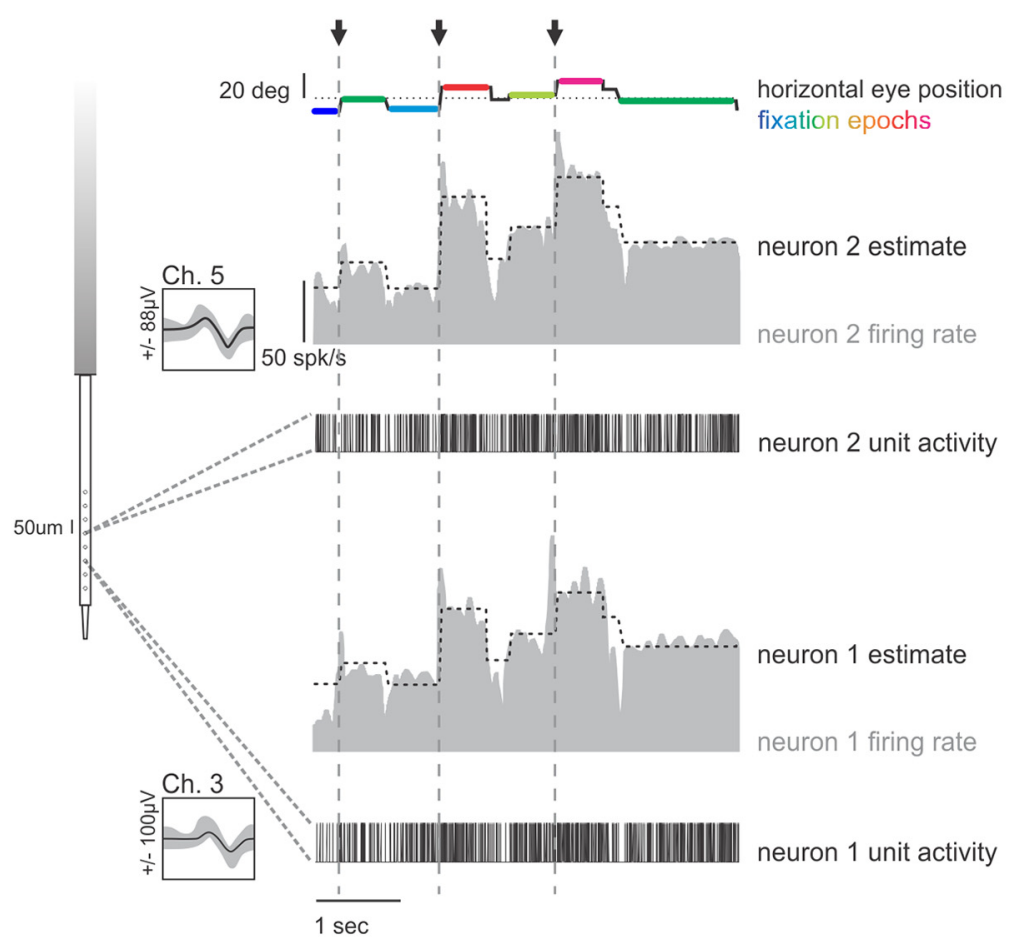

Far pair, 6 spaces apart

\section{B1}

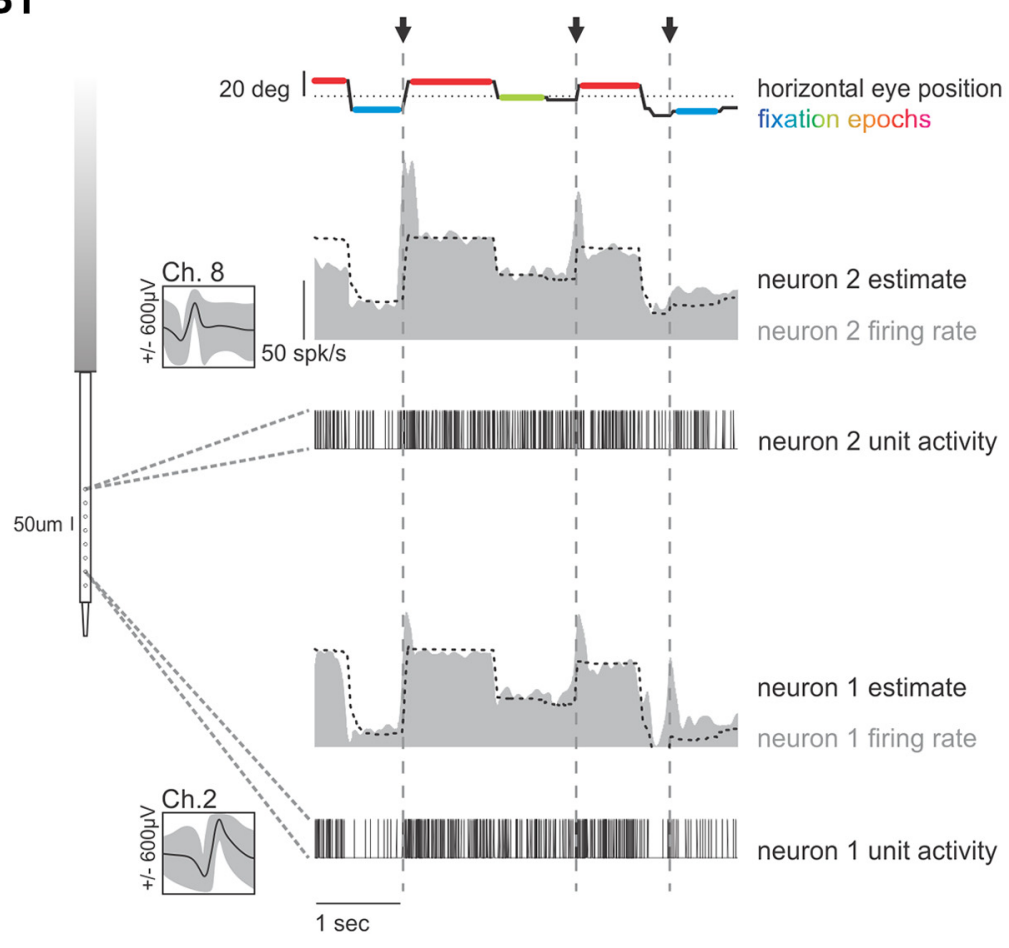

A2

\section{Eye position sensitivity}
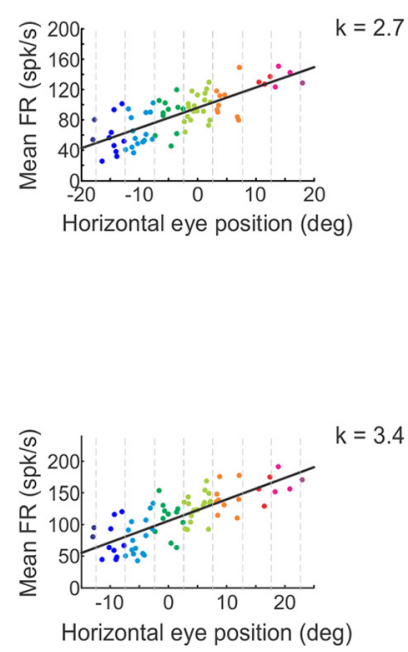

B2

Eye position sensitivity
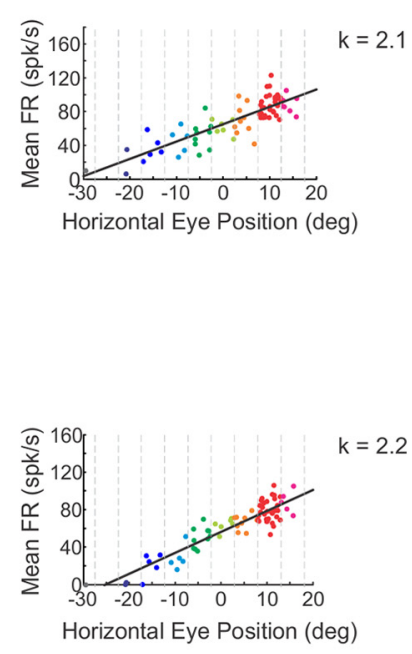

Figure 1. A, Example pair of burst-tonic neurons recorded on channels 3 and 5 of the LMA, i.e., near to one another. $\boldsymbol{A}$, Firing rate and unit activity from an example pair of burst-tonic neurons recorded on channels 3 and 5 of the LMA (left) during spontaneous eye movements (top line). Arrows and dashed vertical lines indicate occurrence of ipsilateral saccades. Dotted line shows correspondence between firing rate and eye position. $A 2$, Firing rate versus horizontal eye-position curves for each of the two neurons, both exhibiting significant positive slopes. $B$, Example pair of burst-tonic neurons recorded on channels 2 and 8 of the LMA, i.e., far from one another. $\boldsymbol{B} 1, \boldsymbol{B 2}$, same as $\boldsymbol{A} \mathbf{1}$ and $\boldsymbol{A} \mathbf{2}$. Color scale corresponds to eye position during fixations, as binned in $\boldsymbol{A} \mathbf{2}$ and $\boldsymbol{B} 2$. Ch, Channel; FR, firing rate. 
A

A Signal correlations

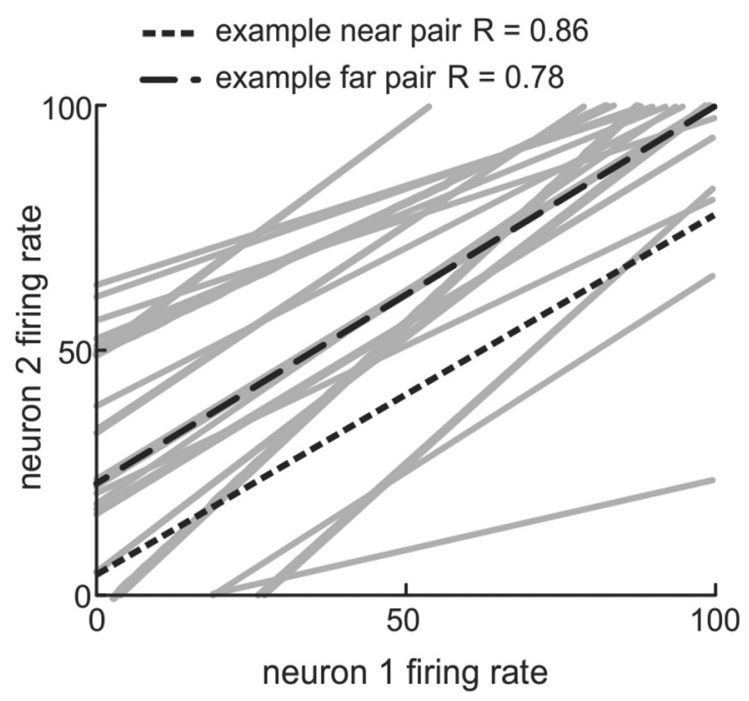

B

\section{Cross correlation functions}

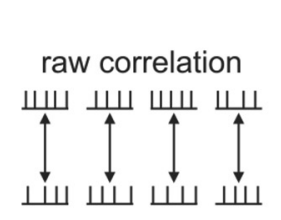

$$
\begin{aligned}
& \text { shuffled correlation } \\
& \text { ("expected") } \\
& \text { ШШ ШШШ } \begin{array}{l}
\text { unit } 1 \\
\text { activity }
\end{array} \\
& \text { 山لّ }
\end{aligned}
$$

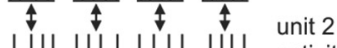

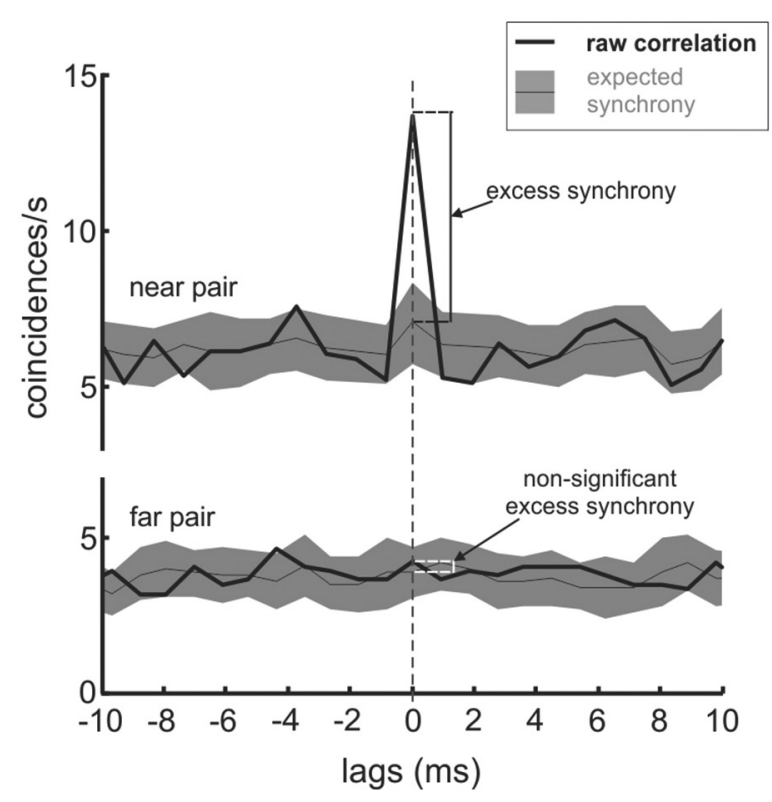

Figure 2. A, The two burst-tonic neurons in each pair show strong correspondence between their firing rates, as anticipated by their similarly positive eye-position tuning. The two example pairs from Figure 1 are highlighted. $\boldsymbol{B}$, Measurements of synchrony are obtained by computing cross-correlations between the unit activities of the two neurons in a pair. Top, Raw correlations are between simultaneously recorded epochs; shuffled correlations are between reordered (non-simultaneous) epochs and are used to obtain an expected range of synchrony. Bottom, We define excess synchrony as the amount by which the raw correlation exceeds the average expected synchrony.
This value is used to define excess synchrony reported in Results below (Fig. $2 B$, bottom). If the average raw cross-correlation fell outside $2 \mathrm{SDs}$ of the average shuffled cross-correlation, i.e., if the value $C_{1}(0)-\left[C_{2}(0)\right.$ $+2 \times \mathrm{SD}]$ was nonzero, we reported significant excess synchrony.

Unless noted otherwise, results are reported as mean \pm SD. Statistical significance was determined by Student's $t$ test.

\section{Results}

In this study, we simultaneously recoded from pairs of bursttonic neurons in the nucleus prepositus ( $n=29$ pairs) to better understand whether and how interneuronal synchrony across the population plays a role in encoding eye position. First, we demonstrated the presence of zero-lag correlations between the firing of burst-tonic neurons that exceeded the level of correlation expected as a result of the highly correlated firing rates of these neurons (i.e., excess synchrony). Next, we considered spike trains recorded during ocular fixations at different positions to determine whether there is a relationship between the magnitude of this excess synchrony and the functional output of the nucleus prepositus (i.e., eye position). Finally, we quantified differences in correlations for pairs of nearby versus distant neurons to investigate how connectivity between the neurons affects the magnitude of excess synchrony.

\section{Pairs of prepositus neurons exhibit excess synchrony}

We initially confirmed that the response of each individual neuron was consistent with that of the burst-tonic neurons shown previously to constitute the majority of neurons within the nucleus prepositus (McFarland and Fuchs, 1992; Cullen et al., 1993; Sylvestre et al., 2003; Dale and Cullen, 2013). Figure 1 shows the firing rates and corresponding spike trains from two example pairs of simultaneously recorded neurons: one pair was recorded on LMA channels 3 and 5 (i.e., $\sim 100 \mu \mathrm{m}$ apart, near pair; Fig. $1 A$ ), and the other pair was recorded on channels 2 and 8 (i.e., $\sim 300 \mu \mathrm{m}$ apart, far pair; Fig. $1 B$ ). All four neurons were typical in that they fired a burst of activity immediately before ipsilateral saccades (Fig. 1, arrows), and that their tonic firing rate encoded eye position during ocular fixations (Fig. 1A1,B1, colored fixation epochs). We quantified the sensitivity of each neuron to eye position as the slope of the best-fit line through a scatter plot showing the average firing rate versus eye position across all fixations. As expected based on previous analyses of burst-tonic neurons, the two neurons in the example near pair (Fig. 1A2) and the two neurons in the example far pair (Fig. 1B2) were each characterized by significant, positive eye-position sensitivity [population average, $k=2.5 \pm 1.0($ spikes $\left./ \mathrm{s}) /^{\circ}\right]$.

Because they share a linear relationship to eye position, the firing patterns of any two burst-tonic neurons should, probabilistically, be highly correlated. Figure $2 A$ shows the trend of the mean firing rate of neuron 2 versus the mean firing rate of neuron 1 across fixation epochs for each pair in our sample. Indeed, as expected from their positive eye-position sensitivities shown in Figure 1, $A 2$ and B2, both of the example pairs exhibited significant positive correlation between the changes in their firing rates with eye position. Across our population, all recorded pairs had significant positive linear regressions between their firing rates during fixations (Fig. $2 A$; on average, slope of $0.64 \pm 0.41, R^{2}=$ $0.63 \pm 0.26, p \leq 0.03)$.

We next computed cross-correlations between the spike trains of these same pairs of neurons to determine whether their firing further showed near-zero-lag correlations, indicative of synchrony. Figure $2 B$ illustrates the raw cross-correlation between the two example pairs of neurons (Eq. 1; Fig. 2B, top, left) and an 
A

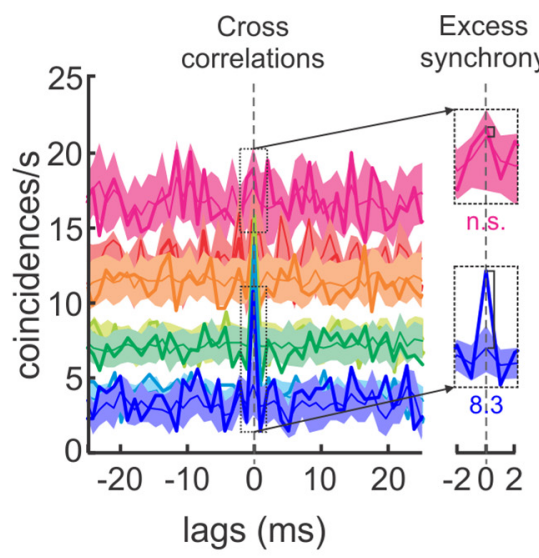

C

Average expected correlation across pairs

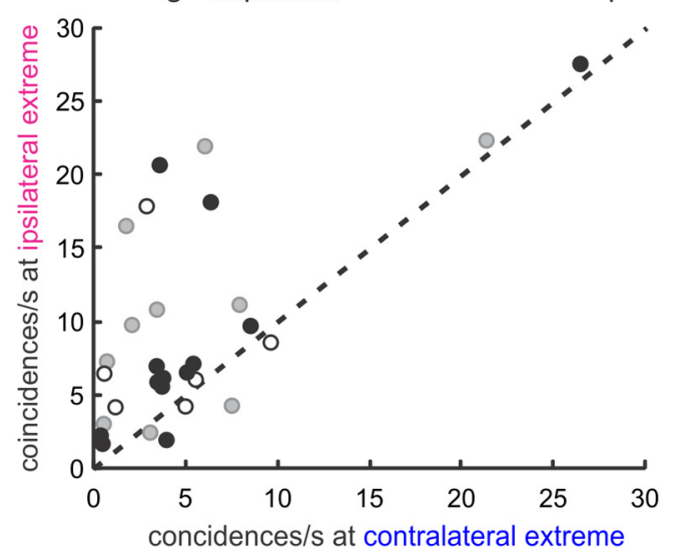

B Far pair

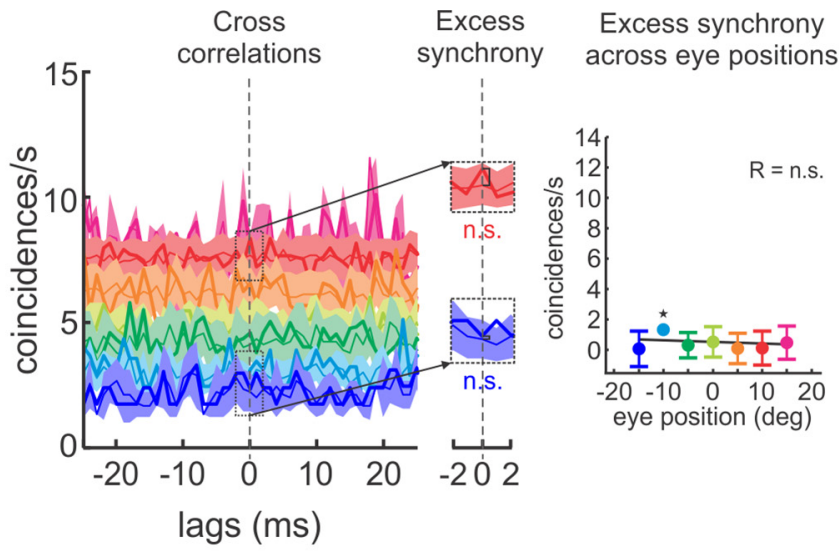

D

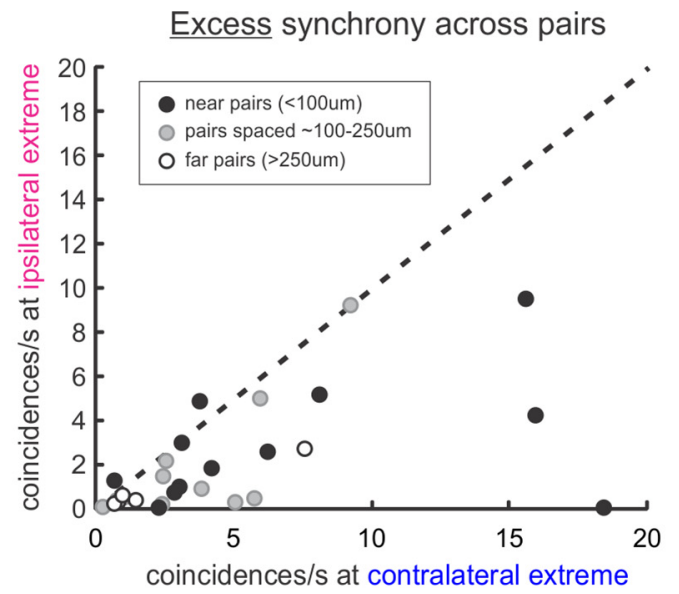

Figure 3. A, Average cross-correlations binned and color coded by eye position for the same example near pair as shown in Figures 1 and 2 . The predictable level of correlation between the two neurons increases from contralateral (blue) to ipsilateral (red) eye positions. Magnified $x$-axis highlights the presence of excess synchrony for example eye positions (expected, 3.2 and 16.2 coincidences/s; excess, 8.3 and 0.8 coincidences/s). Inset, Average excess synchrony over all epochs in each fixation bin shows a decreasing trend across eye positions. Error bars show SEM, and stars indicate magnitudes significantly different from zero. B. Corresponding plots for the same example far pair as shown in Figures 1 and 2 depict the anticipated increase in expected correlations from contralateral to ipsilateral eye positions ( 1.6 and 6.8 coincidences/s) but generally nonsignificant positive excess synchrony ( 0.08 and 1.4 coincidences/s), quantified for all eye positions in the inset. C, Sample summary of the trend in expected correlations: pair by pair, the average magnitude of expected correlations is greater for the most ipsilateral fixation epochs compared with the most contralateral fixation epochs for near pairs (black circles), far pairs (white circles), and pairs at intermediate spacing (gray circles, dashed line shows unity). D, Sample summary of the trend in excess correlations: pair by pair, the average magnitude of excess correlations is greater for the most contralateral fixation epochs compared with the most ipsilateral fixation epochs. Far pairs (white circles) exhibit the lowest excess synchrony overall.

estimate of their expected cross-correlation (shuffled; Eq. 2; Fig. $2 B$, top, right) from responses recorded during ocular fixation with the eyes centered. We typically observed a positive peak in the raw correlation at zero lag and hereafter use the value of the correlation measured at this lag as a measure of synchrony. For the majority of pairs in our sample (17 of 29), the crosscorrelation of the raw spike trains $\left[C_{1}(0)\right.$ in Materials and Methods] significantly exceeded that of the shuffled spike trains $\left[C_{2}(0)\right.$ in Materials and Methods]. Notably, this difference (i.e., excess synchrony) was significant for the example near pair but not the example far pair (Fig. 2B, bottom). We further discuss this difference below.

\section{Synchrony between prepositus neurons is greatest for contralateral eye positions}

To determine whether there is a relationship between the magnitude of excess synchrony and the functional output of the nucleus prepositus (i.e., eye position), we next quantified correlations between spike trains for each pair of burst-tonic neurons during ocular fixation at different positions. Figure $3 A$ shows crosscorrelations computed for the example near pair of burst-tonic neurons at different eye positions (note that ocular fixations spanning from contralateral to ipsilateral are represented by colors ranging blue to red). First, as anticipated from the parallel increases in firing rate for the two neurons as a function of increasing ipsilateral eye position (Fig. $2 A$ ), we found that the average strength of the raw correlation systematically increased (i.e., from blue to red). Importantly, however, although the raw correlation between the two neurons typically fell within the expected range of correlation, most correlograms also depict large excess peaks in the raw correlation near zero lag. Notably, we found that, in contrast to expected correlation, the level of excess synchrony for the example near pair steadily decreased with increasing ipsilateral eye position. This trend is apparent for the example correlations shown in the magnified traces in Figure $3 \mathrm{~A}$. Specifically, we found that the average excess synchrony was 
Contralateral

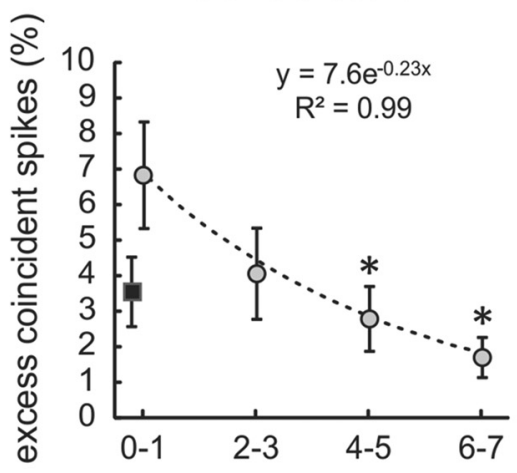

Central

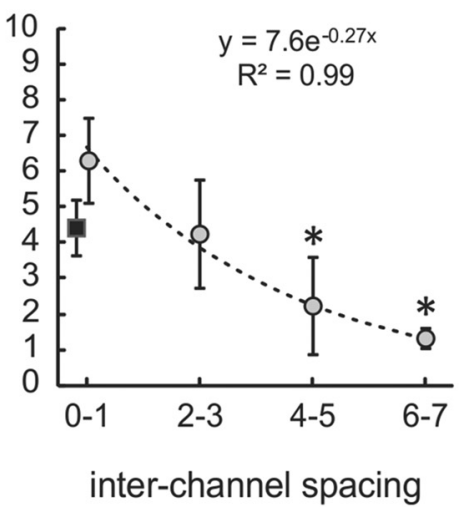

Ipsilateral

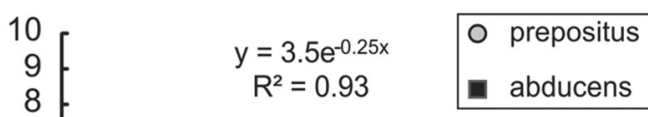

Figure 4. Burst-tonic neuron pairs (circles) grouped by the distance between them (i.e., number of spaces between the LMA channels on which they were isolated). There is an exponential decay in average excess synchrony measured for contralateral (left), central (middle), and ipsilateral (right) fixation epochs with increasing spacing. ${ }^{*} p<0.05$, significant difference from the average for pairs spaced zero to one channels apart. Squares represent corresponding data from seven pairs of abducens motoneurons. Abducens neurons show no change from contralateral to central to ipsilateral fixations (average excess synchrony, $3.6 \pm 1.0,4.4 \pm 0.8$, and $3.7 \pm 0.8 \%$, respectively), which contrasts with the population of nucleus prepositus neurons (average excess synchrony, $7.2 \pm 1.5,6.3 \pm 1.7$, and $3.2 \pm 0.9 \%$, respectively). Note that the level of excess synchrony is only comparable between the two sample populations for ipsilateral fixations ( $p=0.19)$. Error bars Show SEM.

maximal for the most contralateral (dark blue) fixations and then systematically decreased over the entire range of eye positions tested (Fig. 3A, inset). A comparable analysis of our example far pair also produced a predictable increase in cross-correlations from activity during contralateral fixations to ipsilateral fixations (Fig. 3B, blue to red shaded bands). Interestingly, however, the example far pair did not reliably exhibit significant peaks in the raw correlations near zero lag (Fig. 3B, magnified example traces). Accordingly, as shown in the inset of Figure $3 B$, there was not a significant linear relationship between excess synchrony and eye position for the far neuron pair. This apparent distinction will be addressed in the next section.

Figure 3, $C$ and $D$, summarizes the above findings for all of the pairs in our population. Specifically, when we plotted the average expected correlation between each pair at the most ipsilateral fixation position $\left(15\right.$ to $20^{\circ}$ ) versus that at the most contralateral fixation positions $\left(-15\right.$ to $20^{\circ}$ ), nearly all data fell above the unity line (Fig. 3C). This demonstrates that, as predicted by Figure $2 A$, there is a positive relationship between expected correlations and ipsilateral eye position because the firing rates of two neurons both increase as a function of increasing ipsilateral eye position. In contrast, a corresponding pair-by-pair plot of average excess synchrony (Fig. 3D) depicts most data points below the unity line, indicating a negative relationship between excess synchrony and ipsilateral eye position. We note that, although the distributions of the expected correlations of near and far pairs of bursttonic neurons overlapped (Fig. 3C), there appeared to be a distinction between their excess synchrony (Fig. 3D). We next sought to address whether or not this was the case.

\section{Nearby prepositus neurons exhibit the greatest excess synchrony}

To quantify how the positive excess synchrony observed between burst-tonic neuron spike trains depended on how far apart they were recorded along the LMA, we computed excess synchrony at three fixation positions (extreme contralateral, centered, and extreme ipsilateral) for pairs grouped as having been recorded zero to one, two to three, four to five, or six to seven channels apart (50 $\mu \mathrm{m}$ spacing). If excess synchrony between nucleus prepositus neurons is caused by shared input, we would expect the spatial extent of excess synchrony to correspond to that of the input. Indeed, we found that, for each representative eye position, the amount of excess synchrony between neurons (computed as a percentage of spikes to facilitate comparison across pairs) decayed exponentially as a function of the increasing distance between them (Fig. 4). This suggests that nearby neurons are more strongly related than those farther apart, which is in agreement with previous suggestions of a chain of neurons in the nucleus prepositus wherein each neuron is connected with its nearest neighbors (Escudero et al., 1992). At the same time, we found that the overall level of excess synchrony across the ensemble of pairs decreased from contralateral to ipsilateral fixations (Fig. 4) as discussed above. Thus, our results establish that burst-tonic neurons in the primate nucleus prepositus exhibit correlated activity that covaries with both the functional output of the nucleus (i.e., eye position) and their anatomical proximity, thereby providing new insight into the functional organization within the nucleus prepositus.

\section{Synchrony between abducens motoneurons does not change with eye position}

We conducted a comparable analysis of seven pairs of abducens motoneurons, each recorded $<100 \mu \mathrm{m}$ apart, to determine whether the presence of localized excess synchrony is common to all neurons that have burst-tonic firing patterns or instead is unique to the nucleus prepositus. In agreement with the encoding of ipsilateral eye position by abducens motoneurons (Fuchs and Luschei, 1970; Robinson, 1970; Sylvestre and Cullen, 1999), we found that all pairs showed significant positive correlations that increased with increasing ipsilateral eye position. Furthermore, the abducens pairs exhibited peaks in their crosscorrelations at zero lag as a result of excess synchrony. Interestingly, however, we saw no significant trend in excess synchrony across eye positions (Fig. 4, black squares), which contrasts with our sample of nucleus prepositus neuron pairs recorded at a similar distance (i.e., near pairs; Fig. 4, distance zero to one). Pairs of abducens motoneurons are only comparable with their nucleus prepositus counterparts for extreme ipsilateral fixations (Fig. 4, right) in which their excess synchrony is the lowest. 


\section{Contralateral fixation}
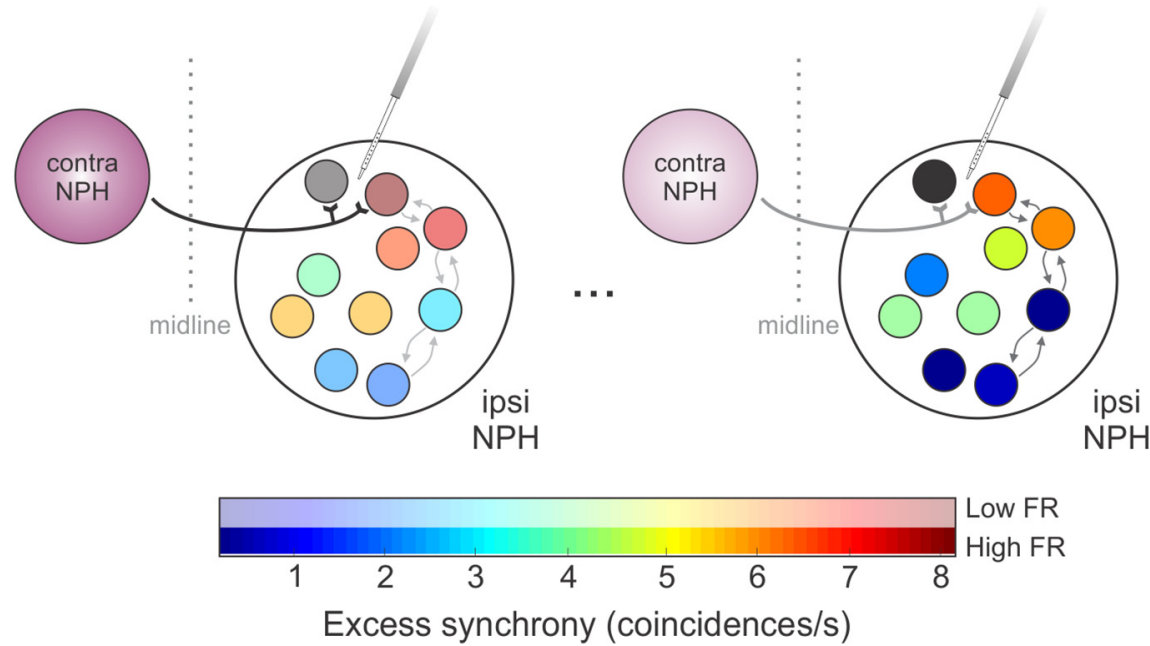

Figure 5. Schematic of the intrinsic connectivity hypothesized to play a role in synchrony within the nucleus prepositus. During extreme contralateral ocular fixation (left), ipsilateral burst-tonic neurons exhibit relatively low firing rates (paler colors) but relatively high excess synchrony (redder color, coded according to correlation with the black neuron). Synchrony could be the result of (1) local projections (small arrows), but the results of the current study are more consistent with (2) common input (thick black line) arising from the contralateral nucleus prepositus, which is firing at its strongest. During extreme ipsilateral fixations (right) when the projection from the contralateral nucleus prepositus is weaker (gray line), ipsilateral burst-tonic neurons exhibit weaker excess synchrony (bluer colors) despite high firing rates (darker colors). contra, Contralateral; FR, firing rate; ipsi, ipsilateral; $\mathrm{NPH}$, nucleus prepositus hypoglossi.

\section{Discussion}

In this study, we used LMAs to simultaneously record ipsilateral pairs of burst-tonic neurons in the primate nucleus prepositus during ocular fixation in the horizontal plane. Analyses were designed to determine whether these neurons exhibit correlations in firing and to investigate what factors influence the level of correlation. Our results demonstrate that pairs of neurons exhibit synchrony at zero lag exceeding that expected from their typical eye-position sensitivity by $\sim 7 \%$ of total spikes. Furthermore, we show that this excess synchrony is greatest when neurons encode more contralateral eye positions and least for more ipsilateral eye positions. When we further considered the ensemble of prepositus neurons, we found that the level of excess synchrony was inversely related to the distance between the two neurons in a pair. In contrast, although on average neighboring abducens motoneurons showed $\sim 4 \%$ excess synchrony, the magnitude did not vary with eye position. The results demonstrated here provide electrophysiological evidence supporting two possible sources for synchrony between burst-tonic neurons in the nucleus prepositus - direct, intrinsic, ipsilateral connections between neurons and shared extrinsic inputs to multiple neuronsboth of which have been described previously through anatomical studies (monkey: Belknap and McCrea, 1988; cat: McCrea and Baker, 1985a,b). Additionally, our findings suggest a common strategy for neural integration in the mammalian nucleus prepositus and its non-mammalian (goldfish: Aksay et al., 2003; zebrafish: Miri et al., 2011) homologs.

\section{Anatomical influences on nucleus prepositus synchrony}

In the present study, our quantification of excess synchrony revealed that pairs of nucleus prepositus burst-tonic neurons fire more coincident action potentials than expected based on probability estimates from their shared linear relationship to eye position. Our observation of a strong correlation at near-zero lag further suggests either direct connections between the two neurons or a common input to both (Perkel et al., 1967). Previous anatomical and electrophysiological studies have provided evidence for each of these two possibilities. First, although to date there is no indication of electrotonic coupling in the nucleus prepositus (Condorelli et al., 2000), intracellular labeling and single-unit characterizations have demonstrated the presence of intrinsic, ipsilateral, excitatory projections between individual neurons (McCrea and Baker, 1985a,b; Delgado-García et al., 1989; Escudero et al., 1992). These direct connections could provide a substrate for synchronizing action potentials within 1 ms (Highstein, 1973) between two nearby neurons. Second, projections from neurons elsewhere in the brainstem provide a shared common input consistent with our observation that neighboring neuron pairs are more synchronized. In particular, the collaterals of excitatory premotor projections from the contralateral vestibular nuclei terminate in the nucleus prepositus over a span of $\sim 200 \mu \mathrm{m}$ (McCrea et al., 1987, their Fig. 10). This range corresponds well with the spatial extent of excess synchrony measured in the present study (Fig. 4). Thus, our findings reveal electrophysiological evidence consistent with shared common inputs to and local synaptic connections between pairs of neurons in the nucleus prepositus.

\section{Nucleus prepositus synchrony and the encoding of eye position}

In our analysis of correlated activity between neuron pairs, we further found that the amount of excess synchrony decreased for encoding ipsilateral versus contralateral eye position (Figs. 3, 4). Theoretically, any external common input could produce such trends in excess synchrony, through either modulation of the input itself or increases in its relative influence as surrounding activity in the nucleus prepositus decreases. Notably, the common input from the vestibular nuclei mentioned above encodes eye position with the same directionality as the recorded bursttonic neurons, which would lead to increased excess synchrony during ipsilateral fixations-a prediction not supported by our results. Moreover, although there is a striking similarity between external inputs to abducens and prepositus (McCrea and Horn, 2006), abducens motoneurons do not exhibit any eye positionrelated changes in excess synchrony (Fig. 4). Thus, we suspect that the behaviorally related synchrony we observe in the prepositus is mediated by intrinsic circuitry. For example, local excitatory connectivity within the prepositus could potentially regulate the influence of external inputs and produce the eye position-dependent trends shown here. Furthermore, there is an important source of common input within the prepositus, namely strong inhibitory projections from the contralateral nucleus prepositus, which arborize within confined areas (McCrea and Baker, 1985b; Escudero and Delgado-García, 1988). Figure 5 shows a schematic of this latter scenario, in which common inhibitory input hypothetically contributes to increasing excess synchrony between nearby neurons. Recall that neighboring neu- 
ron pairs exhibit the least excess synchrony during "on" direction (i.e., ipsilateral) ocular fixation (Figs. 3, 4). Because neural activity in the contralateral nucleus prepositus reverses that of our recorded population, the common drive from the contralateral nucleus prepositus would be minimal ("off") in this condition (Fig. 5, ipsilateral fixation). Similarly, excess synchrony was maximal when recorded neuronal firing rates were weakest and the common input from contralateral burst-tonic neurons would be strongest (Fig. 5, contralateral fixation).

Seung et al. (2000) predicted that continuous output from an ipsilateral population of eye position-encoding neurons produces the most sustained behavior. Based on our current results, we propose that decreased synchrony during ipsilateral eyeposition encoding distributes action potentials in time, resulting in the most constant population output. This idea is further supported by Aksay et al. (2007), whose results suggest that the ipsilateral neuronal population locally performs velocity-to-position integration, whereas gated inhibition between the ipsilateral and contralateral nuclei helps to coordinate the two sides. Specifically, simulations implied that projections from the contralateral nucleus prepositus will provide common, synchronizing input to their target neurons when their own firing is strongest (i.e., during contralateral fixations). Together with the present results, these findings suggest that common inhibitory inputs contribute to successful ipsilateral eye-position encoding through modulated synchrony.

\section{Synchrony in the neural integrator}

Analogous to mammals, fish display eye-movement behaviors requiring neural integration to maintain the position of the eyes (e.g., fixations or slow, smooth movement in response to vestibular stimulation and/or visual scene motion), and the structure that accomplishes this integration, area I, is homologous to prepositus in that it mainly comprises eye position-encoding neurons with a large range of firing rate sensitivities to eye movement (monkey: McFarland and Fuchs, 1992; goldfish: Aksay et al., 2000; Debowy and Baker, 2011; for review, see Joshua and Lisberger, 2014). Paired recordings from area I have provided evidence for synchronous firing between neurons in this structure (Aksay et al., 2003). Notably, consistent with our findings in the primate neural integrator, synchrony between area I neurons is higher during contralateral than ipsilateral fixations. Furthermore, using calcium imaging, Miri et al. (2011) observed graded levels of correlation across the zebrafish oculomotor neural integrator that corresponded to the relative proximity of two neurons. This demonstration of particularly strong correlations between neighboring neurons could reflect direct connections between them (Aksay et al., 2001) or input projections targeting many neurons in a confined area.

Our results from electrophysiological recordings across the primate nucleus prepositus further this existing work by demonstrating that synchrony (i.e., coincident spiking) depends on the distance between neurons. Moreover, the present results not only provide new insight into the intrinsic architecture of the neural integrator in mammals but also validate the use of models built with an understanding of intrinsic connections from data in fish. However, it is important to note that the primate nucleus prepositus comprises more neurons than its fish counterpart and functions over a larger oculomotor range and across a broader scope of oculomotor control (i.e., smooth pursuit and vergence eye movements). Thus, additional studies are required to determine the comparability of circuit dynamics between the primate nucleus prepositus and its fish homologs.
Our finding that the level of synchrony within the mammalian nucleus prepositus correlates with eye position in a manner comparable with that observed in fish suggests a common strategy for the generation of persistent tonic firing. Interestingly, we did not observe the same eye-position dependence in synchrony between abducens motoneurons, which receive direct nucleus prepositus input and also display persistent tonic firing during ocular fixation (Fig. 4). This finding further supports our proposal that the decreased excess synchrony we observed between neighboring nucleus prepositus neurons for contralateral versus ipsilateral fixation is not simply a common feature of neurons that encode eye position but instead arises as a result of the intrinsic architecture of the oculomotor neural integrator.

\section{Conclusions}

In summary, our results demonstrate the presence of excess synchrony in the spike trains of simultaneously recorded burst-tonic neurons in the primate nucleus prepositus. Specifically, we have shown that (1) although firing rate increases for ipsilateral fixations, excess synchrony is reduced, and (2) nearby pairs of neurons exhibit greater excess synchrony than more distant pairs. The excess synchrony we observed could be caused by both direct intrinsic connections between and common input to pairs of neurons. However, we suggest that behaviorally related changes in excess synchrony are likely attributable to shared contralateral inhibitory input among burst-tonic neurons within a limited area. We speculate that the regulation of this input is an important component of maintaining sustained eye-position information that is used by the rest of the brain.

\section{References}

Aksay E, Baker R, Seung HS, Tank DW (2000) Anatomy and discharge properties of pre-motor neurons in the goldfish medulla that have eyeposition signals during fixations. J Neurophysiol 84:1035-1049. Medline

Aksay E, Gamkrelidze G, Seung HS, Baker R, Tank DW (2001) In vivo intracellular recording and perturbation of persistent activity in a neural integrator. Nat Neurosci 4:184-193. CrossRef Medline

Aksay E, Baker R, Seung HS, Tank DW (2003) Correlated discharge among cell pairs within the oculomotor horizontal velocity-to-position integrator. J Neurosci 23:10852-10858. Medline

Aksay E, Olasagasti I, Mensh BD, Baker R, Goldman MS, Tank DW (2007) Functional dissection of circuitry in a neural integrator. Nat Neurosci 10:494-504. CrossRef Medline

Arnold DB, Robinson DA (1997) The oculomotor integrator: testing of a neural network model. Exp Brain Res 113:57-74. CrossRef Medline

Belknap DB, McCrea RA (1988) Anatomical connections of the prepositus and abducens nuclei in the squirrel monkey. J Comp Neurol 268:13-28. CrossRef Medline

Cannon SC, Robinson DA (1985) An improved neural-network model for the neural integrator of the oculomotor system: more realistic neuron behavior. Biol Cybern 53:93-108. CrossRef Medline

Condorelli DF, Belluardo N, Trovato-Salinaro A, Mudò G (2000) Expression of Cx36 in mammalian neurons. Brain Res Brain Res Rev 32:72-85. CrossRef Medline

Cullen KE, Chen-Huang C, McCrea RA (1993) Firing behavior of brain stem neurons during voluntary cancellation of the horizontal vestibuloocular reflex. II. Eye movement related neurons. J Neurophysiol 70:844856. Medline

Dale A, Cullen KE (2013) The nucleus prepositus predominantly outputs eye movement-related information during passive and active selfmotion. J Neurophysiol 109:1900-1911. CrossRef Medline

Debowy O, Baker R (2011) Encoding of eye position in the goldfish horizontal oculomotor neural integrator. J Neurophysiol 105:896-909. CrossRef Medline

Delgado-García JM, Vidal PP, Gómez C, Berthoz A (1989) A neurophysiological study of prepositus hypoglossi neurons projecting to oculomotor and preoculomotor nuclei in the alert cat. Neuroscience 29:291-307. CrossRef Medline 
Delgado-García JM, Yajeya J, Navarro-López Jde D (2006) A cholinergic mechanism underlies persistent neural activity necessary for eye fixation. Prog Brain Res 154:211-224. CrossRef Medline

Escudero M, Delgado-García JM (1988) Behavior of reticular, vestibular and prepositus neurons terminating in the abducens nucleus of the alert cat. Exp Brain Res 71:218-222. Medline

Escudero M, de la Cruz RR, Delgado-García JM (1992) A physiological study of vestibular and prepositus hypoglossi neurones projecting to the abducens nucleus in the alert cat. J Physiol 458:539-560. CrossRef Medline

Fuchs AF, Luschei ES (1970) Firing patterns of abducens neurons of alert monkeys in relationship to horizontal eye movement. J Neurophysiol 33:382-392. Medline

Fuchs AF, Robinson DA (1966) A method for measuring horizontal and vertical eye movement chronically in the monkey. J Appl Physiol 21: 1068-1070. Medline

Galiana HL, Outerbridge JS (1984) A bilateral model for central neural pathways in vestibuloocular reflex. J Neurophysiol 51:210-241. Medline

Goldman MS, Kaneko CR, Major G, Aksay E, Tank DW, Seung HS (2002) Linear regression of eye velocity on eye position and head velocity suggests a common oculomotor neural integrator. J Neurophysiol 88:659665. Medline

Green AM, Meng H, Angelaki DE (2007) A reevaluation of the inverse dynamic model for eye movements. J Neurosci 27:1346-1355. CrossRef Medline

Hayes AV, Richmond BJ, Optican LM (1982) A UNIX-based multiple process system for real-time data acquisition and control. WESCON Conf Proc 2:1-10.

Highstein SM (1973) Synaptic linkage in the vestibulo-ocular and cerebellovestibular pathways to the VIth nucleus in the rabbit. Exp Brain Res 17:301-314. Medline

Joshua M, Lisberger SG (2014) A tale of two species: neural integration in zebrafish and monkeys. Neuroscience. Advance online publication. Retrieved February 11, 2014. doi:10.1016/j.neuroscience.2014.04.048. CrossRef Medline

Judge SJ, Richmond BJ, Chu FC (1980) Implantation of magnetic search coils for measurement of eye position: an improved method. Vision Res 20:535-538. CrossRef Medline

Lopez-Barneo J, Darlot C, Berthoz A, Baker R (1982) Neuronal activity in prepositus nucleus correlated with eye movement in the alert cat. J Neurophysiol 47:329-352. Medline
McCrea RA, Baker R (1985a) Cytology and intrinsic organization of the perihypoglossal nuclei in the cat. J Comp Neurol 237:360-376. CrossRef Medline

McCrea RA, Baker R (1985b) Anatomical connections of the nucleus prepositus of the cat. J Comp Neurol 237:377-407. CrossRef Medline

McCrea RA, Cullen KE (1992) Responses of vestibular and prepositus neurons to head movements during voluntary suppression of the vestibuloocular reflex. Ann N Y Acad Sci 656:379-395. CrossRef Medline

McCrea RA, Horn AK (2006) Nucleus prepositus. Prog Brain Res 151:205230. CrossRef Medline

McCrea RA, Strassman A, May E, Highstein SM (1987) Anatomical and physiological characteristics of vestibular neurons mediating the horizontal vestibulo-ocular reflex of the squirrel monkey. J Comp Neurol 264:547570. Medline

McFarland JL, Fuchs AF (1992) Discharge patterns in nucleus prepositus hypoglossi and adjacent medial vestibular nucleus during horizontal eye movement in behaving macaques. J Neurophysiol 68:319-332. Medline

Miri A, Daie K, Arrenberg AB, Baier H, Aksay E, Tank DW (2011) Spatial gradients and multidimensional dynamics in a neural integrator circuit. Nat Neurosci 14:1150-1159. CrossRef Medline

Moschovakis AK (1997) The neural integrators of the mammalian saccadic system. Front Biosci 2:d552-d577. Medline

Perkel DH, Gerstein GL, Moore GP (1967) Neuronal spike trains and stochastic point processes. II. Simultaneous spike trains. Biophys J 7:419_ 440. CrossRef Medline

Robinson DA (1970) Oculomotor unit behavior in the monkey. J Neurophysiol 33:393-403. Medline

Robinson DA (1989) Integrating with neurons. Annu Rev Neurosci 12:33-45. CrossRef Medline

Seung HS, Lee DD, Reis BY, Tank DW (2000) Stability of the memory of eye position in a recurrent network of conductance-based model neurons. Neuron 26:259-271. CrossRef Medline

Sylvestre PA, Cullen KE (1999) Quantitative analysis of abducens neuron discharge dynamics during saccadic and slow eye movements. J Neurophysiol 82:2612-2632. Medline

Sylvestre PA, Choi JT, Cullen KE (2003) Discharge dynamics of oculomotor neural integrator neurons during conjugate and disjunctive saccades and fixation. J Neurophysiol 90:739-754. CrossRef Medline

von Holst E, Mittelstaedt, H (1950) Das Reafferenzprinzip. Naturwissenschaften 37:464-476. 